\title{
Amiloidosis cardíaca: a propósito de un caso
}

\author{
Pablo Antileo, Sebastián Herrera, José Luis Winter, Ricardo Baeza, Alejandro Paredes.
}

División de Enfermedades Cardiovasculares, Pontificia Universidad Católica de Chile.

Resumen: La amiloidosis corresponde a un conjunto de enfermedades que tienen en común el depósito de amiloide en uno o más órganos. El hallazgo típico del compromiso cardíaco secundario a la amiloidosis es la presencia de insuficiencia cardíaca rápidamente progresiva. La historia natural de esta enfermedad en ausencia de tratamiento es de un rápido compromiso con alta mortalidad.

Reportamos el caso de una paciente con esta enfermedad, describiendo su historia, manejo y seguimiento. 


\section{Case report: Cardiac Amyloidosis}

Abstract Cardiac amyloidosis refers to a set of diseases characterized by amyloid deposit in one or more organs. The typical finding of cardiac involvement secondary to amyloidosis is the presence of rapidly progressive heart failure. The natural story of this disease in the absence of treatment leads to rapid deterioration with a high mortality rate We report the case of a patient with amyloidosis and cardiac involvement, describing the clinical history, management and follow up.

Key Words: amyloidosis, cardiac amyloidosis, cardiomyopathy.
Introducción: La amiloidosis corresponde a un conjunto de enfermedades que tienen en común el depósito de amiloide - fibrillas insolubles de origen proteico - en uno o más órganos. Existen más de 23 diferentes precursores de proteínas que pueden formar amiloide en el ser humano ${ }^{1}$. Las fibrillas miden 7.5 a $10 \mathrm{~nm}$ y son resultado de un error en el plegamiento proteico. El hallazgo característico del depósito de amiloide, es su reacción con tinción rojo Congo, produciendo un color verde manzana a la visualización con luz polarizada ${ }^{2}$.

Es una enfermedad rara, con una incidencia de 8 casos por millón de habitantes por año ${ }^{3}$.

La amiloidois puede ser localizada o sistémica y es actualmente clasificada según el tipo de precursor proteico ${ }^{4}$.

La amiloidosis primaria (AL) es más frecuente en países desarrollados, diagnosticándose 2000 a 2500 nuevos casos cada año en EEUU ${ }^{5}$. Consiste en proteínas derivadas de cadenas livianas de inmunoglobulinas monoclonales, que pueden estar asociadas a mieloma, linfoma o macroglobulinemia, pero en la mayoría de los casos corresponden a gamapatías monoclonales "benignas". Se diagnostican en personas sobre los 50 años, y el compromiso cardíaco es frecuente, pero su manifestación aislada se produce en menos del $5 \%$.

La amiloidosis adquirida (AA), conocida anteriormente como amiloidosis secundaria, es más frecuente en países en desarrollo, la infiltración miocárdica es rara y está frecuentemente asociado a artritis reumatoide, espondiloartropatías, bronquiectasias y tuberculosis. Existen otros tipos de amiloidosis como la senil y la familiar, esta última 
asociada en la mayoría de los casos a mutación de la transtirretina, cuyas manifestaciones clínicas más importantes son la polineuropatía y el compromiso cardíaco ${ }^{6}$.

\section{Caso Clínico}

Mujer de 56 años, con antecedentes de síndrome de apnea obstructiva del sueño y tabaquismo de 10 paquetes-año. Presenta cuadro de 1 año de evolución caracterizado por fatigabilidad, baja de peso (20 kilos), disnea progresiva hasta alcanzar capacidad funcional III, ortopnea, edema de extremidades inferiores y orina espumosa. Sin historia de angor ni síncope.

Al examen físico presentaba presión arterial de 115/69 y frecuencia cardíaca de $60 \mathrm{lpm}$, destacaba macroglosia e ingurgitación yugular (PVC estimada en 11-12 cm de H20), examen cardíaco normal, crepitaciones en ambas bases pulmonares y extremidades inferiores con edema simétrico bilateral importante. Pulsos arteriales presentes, simétricos.

Hemograma sin alteraciones en sus series, VHS $6 \mathrm{~mm} /$ $\mathrm{hr}$, creatinina de $2,8 \mathrm{mg} / \mathrm{dl}$, hiponatremia de $134 \mathrm{mEq} / \mathrm{L}$, albúmina de $3,2 \mathrm{mg} / \mathrm{dl}$ y pruebas tiroideas normales. Las troponinas fueron negativas, pero los niveles de proBNP estaban elevados $(7983 \mathrm{ng} / \mathrm{ml})$. El Electrocardiograma (Figura 1) presentaba onda QS en V1-V3, complejos de bajo voltaje y alteraciones inespecíficas de la repolarización ventricular.

El Ecocardiograma de superficie (Figura 2) evidenció dilatación biauricular, ventrículo izquierdo no dilatado (43/37 $\mathrm{mm})$ con paredes marcadamente engrosadas $(19 / 20 \mathrm{~mm})$, con ecogenicidad aumentada y fracción de eyección de $55 \%$; ventrículo derecho también hipertrófico con función
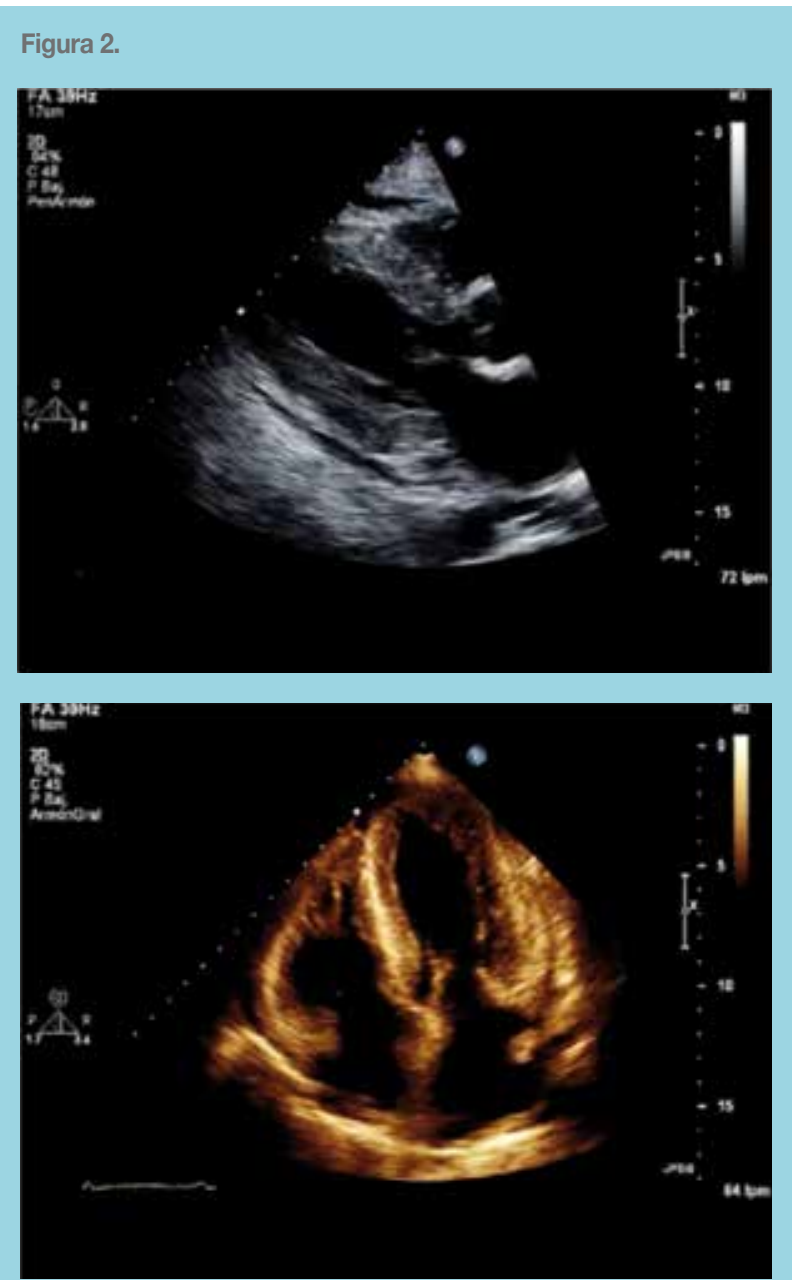

Figura 2. Ecocardiograma de Superficie. Marcado engrosamiento miocárdico de ambos ventrículos, con aumento de ecogenicidad miocárdica y aspecto de "patrón granular". Además, dilatación biatrial y derrame pericárdico.

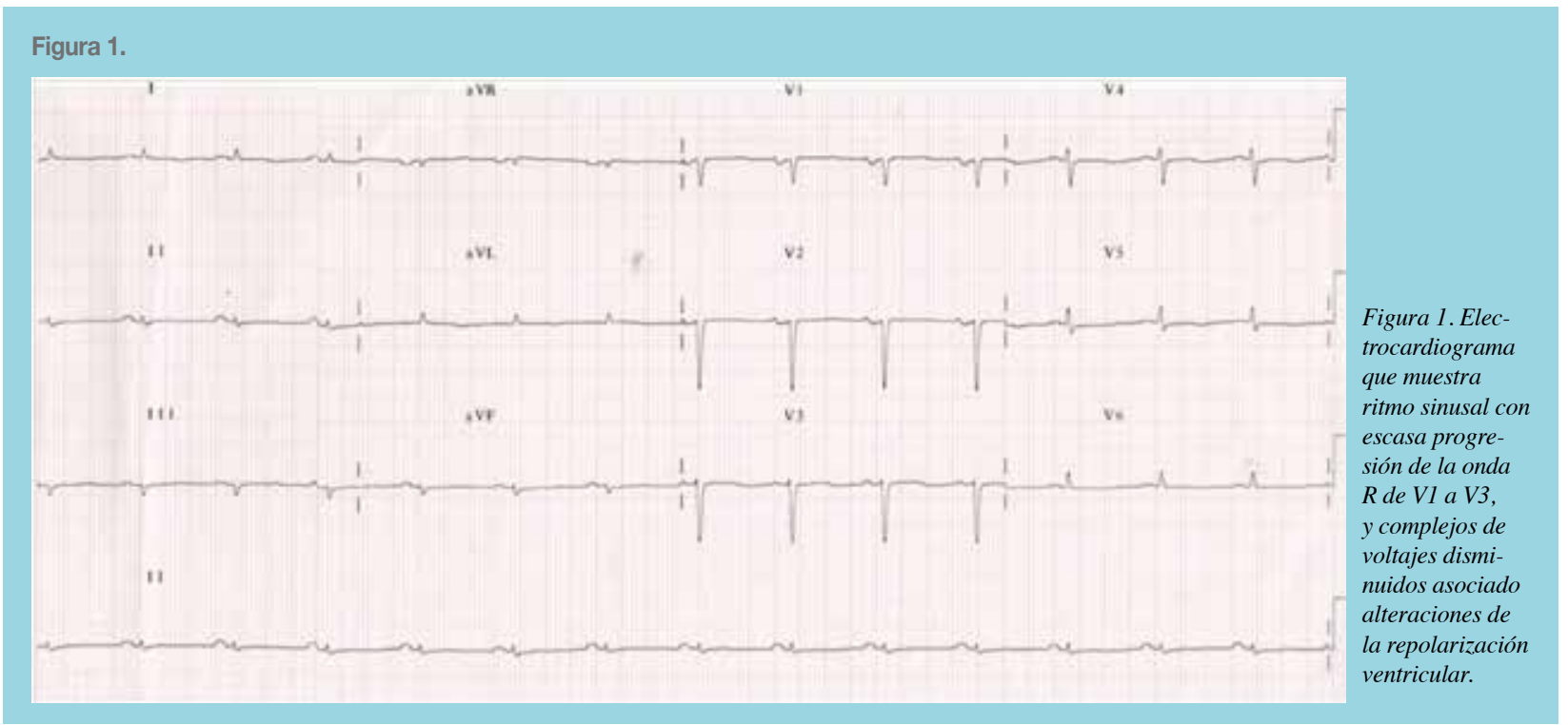


Figura 3.
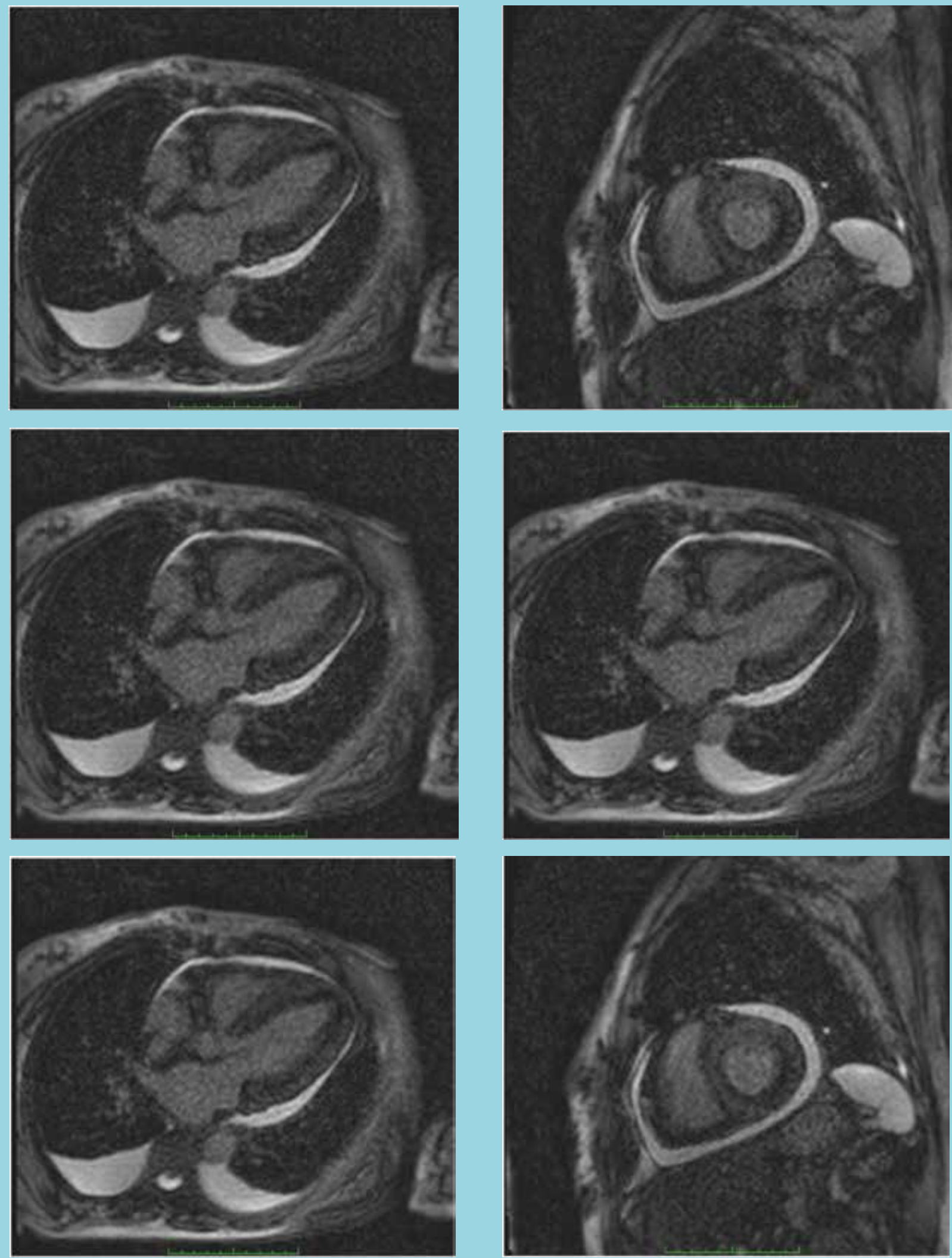
sistólica en rango normal-bajo, insuficiencia mitral mínima y patrón de llenado pseudonormalizado, insuficiencia tricuspídea excéntrica leve a moderada y presión sistólica de arteria pulmonar de $45 \mathrm{mmHg}$. Además, leve a moderado contenido pericárdico.

Ante la sospecha de miocardiopatía infiltrativa se complementa el estudio con Resonancia magnética cardíaca (Figura 3), que mostró engrosamiento miocárdico ventricular difuso mayor a izquierda con refuerzo tardío transmural al gadolinio, compatible con la hipótesis mencionada.

Se continuó el estudio con electroforesis de proteínas en sangre que demostró hipogammaglobulinemia residual y en orina mostrando proteinuria mixta. Inmunofijación de proteínas en suero y orina mostraron componente monoclonal de cadena liviana lambda.

El mielograma mostró hiperplasia global de la médula ósea. En biopsia de médula ósea se observó infiltrado celular atípico con patrón de crecimiento intersticial con células plasmáticas atípicas, identificándose sustancia amorfa de tipo amiloidea en tejido conectivo y algunos vasos con dicroísmo al rojo Congo y resistente al permanganato. El estudio inmunohistoqímico mostró inmunoreactividad para CD138, predominante para cadenas ligeras kappa, linfocitos $\mathrm{T}$ y B politípicos inmunoreactivos para CD3 y CD20, respectivamente. Estos hallazgos fueron compatibles con neoplasia de células plasmáticas con depósito de sustancia amiloidea.

Con los resultados mencionados y el cuadro objetivado de insuficiencia cardíaca, se concluyó como diagnóstico Amiloidosis AL con compromiso cardíaco decidiendo no realizar biopsias adicionales en otros tejidos, iniciando tratamiento con melfalán y dexametasona, manteniendo terapia con nevibolol, furosemida y espironolactona, las cuales se habían iniciado durante hospitalización.

En test de caminata a los 9 meses de seguimiento logra caminar $234 \mathrm{~m}$. A los 14 meses de seguimiento continúa con disnea a pequeños esfuerzos y al examen con presión arterial bien controlada, FC de 90 latidos por minuto, con ingurgitación yugular mínima, sin cambios en la auscultación cardíaca, sin congestión pulmonar y con leve edema periférico.

\section{Discusión}

En este caso se logró llegar al diagnóstico de Amiloidosis tipo AL ante la sospecha de una miocardiopatía infiltrativa por la historia de Insuficiencia cardíaca, hallazgos al examen físico y a la ecocardiografía.

Es importante destacar, en el cuadro clínico de Amiloidosis, que se trata de una enfermedad multisistémica; la mayoría de los pacientes presenta compromiso de otros parénquimas al momento del diagnóstico, que pueden coexistir con la afectación cardíaca, en este caso destacaba la baja de peso significativa de la paciente. La proteinuria es el hallazgo asociado más frecuente, en algunos casos con síndrome nefrótico e insuficiencia renal crónica (presente en más del 50\% de los casos). Otros órganos frecuentemente comprometidos son el sistema nervioso periférico, autónomo, hígado y $\operatorname{piel}^{7}$.

La macroglosia, presente en el caso clínico, es un hallazgo casi patognomónico de amiloidosis AL, en ausencia de acromegalia y mixedema, ocurriendo sólo en el $10 \%$ de los casos; el púrpura periorbitario ocurre como resultado de la fragilidad capilar, secundario a la infiltración por material amiloideo, además de la captación de factores de coagulación como el factor X. Dentro de las manifestaciones neurológicas pueden presentar neuropatía periférica, sin comprometer el sistema nervioso central, pudiendo existir síndrome del túnel carpiano, polineuropatía sensitiva o motora y neuropatía autonómica, manifestándose con hipotensión postural y dismotilidad gastrointestinal. Otros hallazgos son la piel gruesa y cérea por infiltración, hemorragia digestiva y compromiso de glándulas endocrinas por infiltración, con hipotiroidismo e insuficiencia suprarrenal.

El hallazgo típico del compromiso cardíaco secundario a la amiloidosis es la presencia de insuficiencia cardíaca rápidamente progresiva ${ }^{2}$. La fracción de eyección generalmente es normal o discretamente disminuida. La falla cardíaca suele ser global, con predominio del compromiso derecho, presentando edema periférico y ascitis en casos avanzados 8,9 .

El proceso diagnóstico comienza demostrando la presencia de amiloidosis por medio de confirmación histológica. El método clásico muestra fibrillas de amiloide de color "verde manzana" con el uso de luz polarizada. En pacientes con compromiso sistémico se obtiene buen rendimiento con tejido de mucosa rectal, glándulas salivales menores o grasa subcutánea, a través de procedimientos poco invasivos con sensibilidad cercana al $80-85 \%$. En caso de entidades más localizadas se debe obtener muestra del órgano comprometido. Luego de confirmar el diagnóstico se debe demostrar la presencia de gammapatía monoclonal a través de electroforesis de proteínas o inmunofijación de proteínas, ésta última con mejor rendimiento. Otros métodos utilizados actualmente son la medición directa de cadenas livianas en plasma con una sensibilidad cercana al 99\%. Finalmente, debe demostrarse el depósito de cadena liviana en el tejido infiltrado. 
El ecocardiograma juega un rol fundamental con hallazgos distintivos, destacando el engrosamiento parietal ventricular en ausencia de dilatación. La fracción de eyección tiende a ser normal, incluso en casos de insuficiencia cardíaca congestiva ${ }^{10}$. Lo anterior no se debe sólo a disfunción diastólica, sino también al compromiso de miocitos subendocárdicos que son particularmente susceptibles a la infiltración, con rápido deterioro de la contracción longitudinal. El uso de Doppler tisular, con técnicas de strain y strain rate (no efectuadas en este caso) son de particular utilidad, mostrando un compromiso desproporcionado de la función sistólica longitudinal comparado con los valores de la fracción de eyección ${ }^{11}$, 12. El uso conjunto de Doppler transmitral y tisular puede revelar aumento en las presiones de llenado del VI o un patrón de llenado de tipo restrictivo. Otros signos son el engrosamiento de la pared libre del VD, dilatación biauricular marcada, engrosamiento valvular difuso y presencia de derrame pericárdico.

El uso de la cardioRNM en pacientes con amiloidosis cardíaca se ha obtenido en etapas avanzadas de la enfermedad, y su utilidad en fases iniciales aún no ha sido del todo aclarada en la literatura. Se ha descrito un patrón de engrosamiento ventricular con captación difusa del gadolinio, predominantemente subendocárdica en fases tardías ${ }^{13}$, 14 . Este patrón difuso sin afección circunscrita a un territorio coronario, como ocurre en la cardiopatía isquémica, contribuye al momento de hacer el diagnóstico diferencial. El gold standard para demostrar compromiso cardíaco secundario a amiloidosis es la biopsia cardíaca, que se reali- za en la minoría de los pacientes.

Respecto al pronóstico, la historia natural de esta enfermedad en ausencia de tratamiento, es de un compromiso rápidamente progresivo, con una mortalidad cercana a $80 \%$ a dos años ${ }^{15}$. Si existe insuficiencia cardíaca clínica, el pronóstico es malo, con una sobrevida media de 4 a 6 meses. Se ha demostrado que los niveles de troponina y de NT - ProBNP son predictores independientes de sobrevida en pacientes con Amiloidosis AL y se han incorporado en un sistema de etapificación ${ }^{16}$. De esta forma se ha definido Estado I cuando ambos biomarcadores están bajo el valor umbral (troponina cardíaca $\mathrm{T}<0,035 \mathrm{ug} / \mathrm{L}$ y NT- proBNP $<332 \mathrm{ng} / \mathrm{ml}$ ), estadio II cuando uno de los dos está elevado y estadio III cuando ambos estén elevados. La sobrevida promedio es de 26.4, 10.5 y 3.5 meses, respectivamente. Otros factores pronósticos identificados son la fracción de eyección menor al $50 \%$ que disminuye la sobrevida a menos de 3 meses en pacientes en estadio III. El tratamiento de la amiloidosis cardíaca consta de dos partes: por un lado, el tratamiento de los síntomas de origen cardíaco y, por otro, el de la enfermedad de base productora de la proteína amiloide.

Actualmente la decisión de realizar un trasplante cardíaco es compleja, debido a que los pacientes presentan compromiso multiorgánico por el depósito de amiloide, lo cual no les permitiría ser candidatos óptimos. En este grupo de pacientes, los episodios de muerte súbita cardíaca son debidos a actividad eléctrica sin pulso, por lo que el implante de un cardiodesfibrilador deberá limitarse sólo a aquellos que presenten arritmias ventriculares ${ }^{8}$. 


\section{Referencias:}

1. DUBREY SW, HAWKINS PN, FALK RH. Amyloid diseases of the heart: assessment, diagnosis, and referral. Heart. 2011; 97:75-84.

2. FALK RH. Diagnosis and management of the cardiac amyloidoses. Circulation. 2005; 112: 2047-60.

3. BHATTACHARYYA S, DAVAR J, DREYFUS G, CAPLIN ME. Carcinoid heart disease. Circulation. 2007; 116: 2860-5.

4. RAPEZZI C, MERLINI G, QUARTA CC, RIVA L, LONGHI S, LEONE O, et al. Circulation. 2009; 120: 1203-12.

5. SELVANAYAGAM JB, HAWKINS PN, PAUL B, MYERSON SG, NEUBAUER S. Evaluation and management of the cardiac amyloidosis. J Am Coll Cardiol. 2007; 50: 2101-10.

6. FALK RH, COMENZO RL, SKINNER M. The systemic amyloidoses. N Engl J Med. 1997; 337: 898-909.

7. SHARMA N, HOWLETT J. Current state of cardiac amyloidosis. Curr Opin Cardiol. 2013; 28: 242-8.

8. FALK RH, DUBREY SW. Amyloid heart disease. Prog Cardiovasc Dis 2010; 52: 347-361.

9. DUBREY SW, CHA K, ANDERSON J, CHAMARTHI B, REISINGER J, SKINNER M, et al. The clinical features of immunoglobulin light-chain (AL) amyloidosis with heart involvement. QJM 1998; 91: 141-157.

10. TSANG W, LANG RM. Echocardiographic evaluation of cardiac amyloid. Curr Cardiol Rep 2010; 12: 272-276.
11. KOYAMA J, RAY-SEQUIN PA, DAVIDOFF R, FALK RH. Usefulness of pulsed tissue Doppler imaging for evaluating systolic and diastolic left ventricular function in patients with AL (primary) amyloidosis. Am J Cardiol 2002; 89: 1067-1071.

12. KOYAMA J, RAY-SEQUIN PA, FALK RH. Longitudinal myocardial function assessed by tissue velocity, strain, and strain rate tissue Doppler echocardiography in patients with AL (primary) cardiac amyloidosis. Circulation 2003; 107: 2446-2452.

13. AUSTIN BA, TANG WH, RODRIGUEZ ER, TAN C, FLAMM SD, TAYLOR DO, et al. Delayed hyper-enhancement magnetic resonance imaging provides incremental diagnostic and prognostic utility in suspected cardiac amyloidosis. JACC Cardiovasc Imaging 2009; 22:1369-1377.

14. PENNELL DJ, MACEIRA AM. Magnetic resonance imaging in cardiac amyloidosis. JACC Cardiovasc Imaging 2009; 2: 1378-1380.

15. KYLE RA, GERTZ MA, GREIPP PR, WITZIG TE, LUST JA, LACY JA, et al. A trial of three regimens for primary amyloidosis: colchicine alone, melphalan and prednisone, and melphalan, prednisone, and colchicine. N Engl J Med 1997; $336: 1202-1207$

16. DISPENZIERI A, GERTZ MA, KYLE RA, LACY MQ, BURRITT MF, THERNEAU TM, et al. Serum cardiac troponins and N-terminal pro-brain natriuretic peptide: a staging system for primary systemic amyloidosis. J Clin Oncol. 2004; 22: 3751-7. 